\title{
Antioxidant Activity and Cytotoxicity of Medicago sativa L. Seeds and Herb Extract on Skin Cells
}

\author{
Martyna Zagórska-Dziok, Aleksandra Ziemlewska, Zofia Nizioł-Łukaszewska, and Tomasz Bujak
}

\begin{abstract}
In response to the constantly growing demand for high quality cosmetics we observe an increasing interest in products containing natural plant extracts. This article attempts to assess the antioxidant and cytotoxic properties of extracts from alfalfa herb and seeds (Medicago sativa L.). These extracts were obtained using ultrasoundassisted extraction method. The research was carried out on two cell lines: fibroblasts and keratinocytes. The obtained results show that the examined extracts from $M$. sativa $L$. are a source of valuable biologically active agents. Both extracts were characterized by high content of flavonoids and phenolic compounds. Evaluation of antioxidant properties of extracts using DPPH • radical indicated that the alfalfa extracts can efficiently scavenge free radicals. The results of the conducted experiments demonstrated that the M. sativa L. extracts do not only have an inhibitory effect on free radicals existing in the external environment of the cell, but also have the ability to reduce the intracellular reactive oxygen species level, which may contribute to the reduction of oxidative stress inside the cells. Studies performed using cell lines indicated that the tested extracts increase the proliferation and metabolism of skin cells in vitro. The high antioxidant capacity of M. sativa L. extracts may indicate its potential use as a valuable ingredient in the cosmetics and pharmaceutical industries.
\end{abstract}

Keywords: antioxidants; free radicals; cell culture; oxidative stress

\section{Introduction}

The skin is a barrier that separates the body from the external environment. In addition to protecting the body against water loss and infection of microorganisms, it plays an important cosmetic role. ${ }^{1}$ As the most extensive organ in the body, over time the skin shows numerous visible signs of aging. Therefore, many people, especially women, spend a lot of money on cosmetics and pharmaceutical products that help them prevent or partially reverse skin aging. ${ }^{2}$ This aging is caused by both internal and external factors. Internal aging is an unavoidable physiological process that results in thin, fine wrinkles, dry skin, and progressive skin atrophy. In contrast, external aging is caused by external factors such as air pollution, poor nutrition, smoking, and exposure to the sun, which leads to thick wrinkles, laxity and loss of skin elasticity, and rough textured appearance. Moreover long-term exposure to solar ultraviolet (UV) radiation is the basic factor of skin aging called photoaging.,

As is commonly known, skin aging is affected by reactive oxygen species (ROS) such as superoxide anions $\left(\mathrm{O}_{2} \cdot{ }^{-}\right)$, hydroxyl, nitric oxide radicals, and hydrogen peroxide $\left(\mathrm{H}_{2} \mathrm{O}_{2}\right)$. They play an important role in oxidative stress associated with the pathogenesis of various diseases. ${ }^{5}$ Excessive production of free radicals may cause damage to cells and tissues through nonspecific modification and disruption of proteins, phospholipids, and nucleic acids. ROS have a serious negative effect on organelles and cell membranes. Their excess causes peroxidation of membrane lipids and modification of membrane proteins. As a result, the membrane structure is changing and their functions are disturbed. ${ }^{6,7}$

Department of Technology of Cosmetic and Pharmaceutical Products, University of Information Technology and Management in Rzeszow, Tyczyn, Poland.

*Address correspondence to: Aleksandra Ziemlewska, MS, Department of Technology of Cosmetic and Pharmaceutical Products, University of Information Technology and Management in Rzeszow, Kielnarowa 386a, Tyczyn 36-020, Poland, E-mail: aziemlewska@wsiz.rzeszow.pl

(C) Martyna Zagórska-Dziok et al. 2020; Published by Mary Ann Liebert, Inc. This Open Access article is distributed under the terms of the Creative Commons License (http://creativecommons.org/licenses/by/4.0), which permits unrestricted use, distribution, and reproduction in any medium, provided the original work is properly cited. 
Fortunately, well-functioning cells have the ability to defend against the destructive effects of free radicals by the endogenous systems consisting of various enzymes, such as catalase, superoxide dismutase, and glutathione peroxidase. Many scientists have shown that it is possible to reduce the negative influence of free radicals by using appropriate protective agents, that is, antioxidants. Natural antioxidants may be a useful strategy for the prevention of photoaging and oxidative stress closely related to skin diseases. ${ }^{8-11}$ The rich source of natural antioxidants are plants, mainly vegetables, fruits, and herbs, which are a common component of the daily diet. ${ }^{12}$ Plants extracts are currently one of the largest and most valued groups of ingredients used in the pharmaceutical and cosmetics industries. In addition to their strong antibacterial, antifungal, and antiviral activity, they also show strong antioxidant properties. $^{13,14}$ The antioxidant capacity of plant extracts is mainly related to the content of phenols, flavonoids, isoflavonoids, and anthocyanins. Numerous studies have shown a correlation between the content of phenolic compounds and flavonoids in plants and the antioxidant activity of plant extracts. ${ }^{15,16}$ Plant extracts are used in cosmetics mainly to improve their quality and ameliorate functionality. It has been shown that the extracts are much more effective than single compounds due to synergistic interactions between all chemical components. ${ }^{17,18}$ One of the plants containing abundant active ingredients that can be extracted and used as active ingredients in cosmetics preparations is Medicago sativa $\mathrm{L}$.

$M$. sativa $\mathrm{L}$. known as alfalfa is a member of the Fabaceae family, which has a long dietary and medicinal use in traditional herbal medicine in China, India, America, and many Middle Eastern countries for the treatment of a variety of disorders. ${ }^{19}$ In addition, $M$. sativa $\mathrm{L}$. is widely used as animal feed due to the high content of fiber, proteins, minerals, vitamins, chlorophyll, and carotenoids. ${ }^{20}$ It has also been documented that alfalfa is a valuable source of phytochemicals such as alkaloids, amino acids, carotenes, coumarins, digestive enzymes, flavonoids, minerals, nonprotein amino acids, organic acids, phenolic compounds, cyclic polyols, phytoestrogens, phytosterols, polyamines, saponins, and some other volatile organic compounds such as terpenes, furanoids, alcohols, and ketones. The impressive amount of biologically active compounds certainly contributed to the fact that this plant has antioxidant, anti-inflammatory, immunomodulatory, and anticancer properties. ${ }^{21-23}$
The aim of our research was to assess the effect of seed and herb alfalfa extracts on the viability of skin cells, fibroblasts (BJ) and keratinocytes (HaCaT) in vitro. These cells were selected because this article is an attempt to assess the potential use of extracts from this plant in pharmaceutical and cosmetic preparations. Due to the fact that oxidative stress plays an extremely important role in skin condition, this work also includes an assessment of the ability to scavenge free radicals, both in the external environment and inside the cells.

\section{Materials and Methods}

Plant material and extraction procedure

Seeds and herb of M. sativa L. were obtained from the local herbalist. The Plato variety was used in the research. It was produced by Diet-Food, Poland. Then two extracts were made, separately from the herb and alfalfa seeds.

\section{Ultrasound-assisted extraction method}

Extracts from alfaalfa seeds and herb were obtained using ultrasound-assisted extraction method (UAE). UAE was performed according to the method described by Yang et al. in ultrasonic bath (Digital Ultrasonic Cleaner) equipped with time controller. ${ }^{24}$ About $20 \mathrm{~g}$ of M. sativa $\mathrm{L}$. seeds and herb were placed into a glass beaker and $180 \mathrm{~mL}$ a mixture of water and glycerine in the ratio of 80:20 was poured. The mixture was homogenized in room temperature for $50 \mathrm{~min}$ (10 cycles for $5 \mathrm{~min}$ ). Then, obtained extracts were collected and filtered three times through Whatman filter paper No. 10 using a vacuum pump. Extracts were stored in the dark in $4^{\circ} \mathrm{C}$ for further analysis.

\section{Total phenolic content determination}

The total phenolic content in extracts obtained from alfalfa seeds and herb were determined spectrophotometrically by the Folin-Ciocalteu method according to the procedure reported by Singleton et al. with some modifications. ${ }^{25}$ The $300 \mu \mathrm{L}$ of tested extract $(0.25-10 \%)$ and $1500 \mu \mathrm{L}$ of $1: 10$ Folin-Ciocalteau reagent were mixed. After $6 \mathrm{~min}$ of incubation in the dark, $1200 \mu \mathrm{L}$ of sodium carbonate (7.5\%) was added to each sample. After $2 \mathrm{~h}$ of incubation in the dark the absorbance of the tested solutions was measured spectrophotometrically at $\lambda=740 \mathrm{~nm}$ by AquamateHelion (Thermo Scientific). The measurements were carried out at room temperature $\left(25^{\circ} \mathrm{C}\right)$. The total phenolic concentration in analyzed extracts were 
calculated from a gallic acid (GA) calibration curve $(10-100 \mathrm{mg} / \mathrm{mL})$. The estimation of phenolic compounds in the extracts was carried out in triplicate and the results were averaged.

\section{Total flavonoids content determination}

The total flavonoid content (TFC) of analyzed extracts were evaluated using aluminium nitrate nonahydrate according to the procedure reported by Matejić et al. with modifications. ${ }^{26}$ Briefly, $600 \mu \mathrm{L}$ of examined extract solutions $(0.25-10 \%)$ and $2400 \mu \mathrm{L}$ of mixture

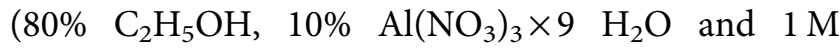
$\mathrm{C}_{2} \mathrm{H}_{3} \mathrm{KO}_{2}$ ) were mixed. After $40 \mathrm{~min}$ of incubation at $25^{\circ} \mathrm{C}$, the absorbance at $415 \mathrm{~nm}$ was measured spectrophotometrically by FilterMax F5 (AquamateHelion). Both types of extracts were used in the analyzes. The total flavonoids concentration in $M$. sativa $\mathrm{L}$. extracts were calculated from a quercetin hydrate $(\mathrm{Qu})$ calibration curve $(10-100 \mathrm{mg} / \mathrm{mL})$ and expressed as quercetin equivalents $(\mathrm{Qu}) / \mathrm{g}$ of extract averaged from three independent measurements.

\section{DPPH Radical Scavenging Assay}

The ability to scavenge free radicals by alfalfa extracts obtained from seed and herb was evaluated using DPPH (2,2-diphenyl-1-picrylhydrazyl) assay according to the method described by Brand-Williams et al. ${ }^{27}$ About $167 \mu \mathrm{L}$ of $4 \mathrm{mM}$ ethanol solution of $\mathrm{DPPH}$ was mixed with $33 \mu \mathrm{L}$ of analyzed samples in different concentrations $(0.25 \%, 0.5 \%, 1 \%, 2.5 \%, 5 \%$, and $10 \%)$. The absorbance was measured at $\lambda=516 \mathrm{~nm}$ in every $5 \mathrm{~min}$ for $30 \mathrm{~min}$. Measurements were made using UV-Vis spectrophotometer Filter Max 5 (Thermo Scientific). DPPH solution mixed with equal volume of distilled water was used as a control. The percentage of DPPH radical scavenging was calculated using the equation:

$\% D P P H \bullet$ scavenging $=\frac{\text { Abs control }- \text { Abs sample }}{\text { Abs control }} \times 100 \%$

\section{Cell culture}

BJ cells (fibroblasts, ATCC ${ }^{\circledR} \mathrm{CRL}-2522^{\mathrm{TM}}$ ) and $\mathrm{HaCaT}$ cells (normal human keratinocytes, ATCC) used in the experiments were obtained from the American Type Culture Collection (Manassas, VA). Cells were maintained in a Dulbecco's modified essential medium (DMEM; Gibco) with L-glutamine, supplemented with $10 \%$ (vol/vol) fetal bovine serum (FBS; Gibco), and $1 \%$ (vol/vol) antibiotics (100 U/mL Penicillin and $1000 \mu \mathrm{g} / \mathrm{mL}$ Streptomycin; Gibco). Fibroblasts were maintained in a Minimum Essential Medium (MEM; Gibco) that contains Earle's salt and L-glutamine, supplemented with $10 \%$ (vol/vol) FBS (Gibco), and 1\% (vol/vol) antibiotics $(100 \mathrm{U} / \mathrm{mL}$ Penicillin and $1000 \mu \mathrm{g} / \mathrm{mL}$ Streptomycin; Gibco). The cultured cells were kept at $37^{\circ} \mathrm{C}$ in a humidified atmosphere of $95 \%$ air and $5 \%$ of carbon dioxide. When the cells reached confluence, the culture medium was removed from the flask (Nest) and cells were rinsed two times with sterile phosphate-buffered saline (PBS; Gibco). The confluent layer was trypsinized using Trypsin/EDTA (Gibco) and then resuspended in fresh medium. The cells were seeded in a 96-well plate bottom (on separate plates for each cell type). After attachment of the $\mathrm{HaCaT}$ and fibroblasts to the bottom of the wells of the plates, cells were incubated with varying concentrations $(0.25 \%, 0.5 \%, 1 \%, 2.5 \%, 5 \%$, and $10 \%)$ of the aqueous glycerin extracts from $M$. sativa $\mathrm{L}$. seeds and herb.

\section{Cell viability assay}

Neutral red uptake assay. The neutral red uptake assay (Sigma Aldrich) was used to assess $\mathrm{HaCaT}$ and fibroblasts viability. This assay is based on the initial protocol described by Borenfreund and Puerner. ${ }^{28}$ It allows to evaluate cell viability and determine accumulation of the neutral red dye in the lysosomes of viable, uninjured cells. The examined cells were placed in 96well plates at a density of $1 \times 10^{4}$ cells/well. After $24 \mathrm{~h}$ of preculture, medium (DMEM or MEM) was aspirated and tested concentrations of $M$. sativa L. UAE seeds and herb extracts (in the range of concentrations from $0.25 \%$ to $10 \%$ ) were added into each well and cultured for another $24 \mathrm{~h}$. The control group was unexposed cells. Following exposure to tested extracts, cells were incubated for $2 \mathrm{~h}$ with neutral red dye $(40 \mu \mathrm{g} / \mathrm{mL})$ dissolved in serum-free DMEM or MEM medium. Then, cells were washed with $\mathrm{PBS}$ and $150 \mu \mathrm{L}$ of destain solution (EtOH/AcCOOH/ $\mathrm{H}_{2} \mathrm{O}_{2}$, $50 \% / 1 \% / 49 \%)$ per well was added. The plates were shaken gently for $10 \mathrm{~min}$ until the neutral red had been extracted from the cells. Neutral red dye uptake was determined by measuring the optical density of the eluted dye at $\lambda=540 \mathrm{~nm}$ in microtiter plate reader spectrophotometer FilterMax F5 (Thermo Fisher). The experiments were performed in triplicates for each extract concentration and presented as percentage of control values (100\%).

Alamar Blue assay. Another test to assess cell viability was the Alamar Blue test (resazurin sodium salt, Sigma, 
R7017). Cells were seeded in transparent 96-well plates at a density of $1 \times 10^{4}$ cells/well with fresh DMEM or MEM medium and exposed to different concentrations $(0.25-10 \%)$ of tested alfalfa seeds and herb extracts for $24 \mathrm{~h}$. The control group was unexposed cells maintained in a DMEM or a MEM medium. After $24 \mathrm{~h}$ of exposure, resazurin solution was transferred into the plates for a final volume of $250 \mu \mathrm{L} /$ well and final concentration of $60 \mu \mathrm{M}$ resazurin. Subsequently, the cells were incubated for $2 \mathrm{~h}$ at $37^{\circ} \mathrm{C}$ in darkness. The absorbance was measured at the wavelength $\lambda=570 \mathrm{~nm}$ using a microplate reader (FilterMax F5; Molecular Devices). The experiments were performed in triplicates for each $M$. sativa $L$. extracts concentration. Results were expressed as a percentage of the viability of the control sample versus the control (100\%).

\section{Detection of intracellular ROS level}

To assess the ability of the analyzed $M$. sativa $\mathrm{L}$. extracts to change the intracellular level of ROS in $\mathrm{HaCaT}$ and fibroblast cells, the fluorogenic dye $\mathrm{H}_{2}$ DCFDA was applied. Analogous to the tests evaluating cell viability, the analyzed cells were seeded in 96 -well plates at a density of $1 \times 10^{4}$ cells per well and initially cultured before the experiment for $24 \mathrm{~h}$. In the next step, the culture medium was removed and $10 \mu \mathrm{M} \mathrm{H}_{2} \mathrm{DCFDA}$ (Sigma Aldrich) in serum-free medium (DMEM or MEM for HaCaT and fibroblasts, respectively) was added. Cells were incubated with $\mathrm{H}_{2}$ DCFDA for $45 \mathrm{~min}$ before extracts treatment. Subsequently, $\mathrm{HaCaT}$ and fibroblast cells were exposed into different glycerin-water extract concentrations $(0.25 \%, 0.5 \%, 1 \%, 2.5 \%, 5 \%$, and $10 \%)$. Cells treated with $1 \mathrm{mM} \mathrm{H}_{2} \mathrm{O}_{2}$ were used as positive control. The $2^{\prime}, 7^{\prime}$-dichlorofluorescein (DCF) fluorescence was monitored every $30 \mathrm{~min}$ for a total $90 \mathrm{~min}$ using a microplate reader FilterMax F5 (Thermo Fisher) at maximum excitation of $\lambda=485 \mathrm{~nm}$ and emission spectra of $\lambda=530 \mathrm{~nm}$.

\section{Statistical analysis}

Obtained values were presented as a mean \pm SD. Significant differences between obtained values were analyzed using StatSoft, Statistica 9.0 using one-way analysis of variance and Tukey's test. Differences were considered significant when $p<0.05$. Statistically significant differences are marked on the charts with letters. Statistically significant differences were marked with different letters.

\section{Results}

The presence of only a few studies comparing active compounds in different parts of alfalfa contributed to the undertaking of studies aimed at assessing the antioxidant properties of water-glycerin extracts from seeds and herbs of $M$. sativa $L$. The study also determined the biological activity of these extracts using in vitro model cell lines.

The article attempts to determine the total content of phenols (TPC) and the TFC. The amounts of these compounds were determined from calibration curves of gallic acid $(y=0.0046 \mathrm{x}+0.0452, \mathrm{R} 2=0.9989)$, and quercetin $(y=0.0153 \mathrm{x}-0.0053, \mathrm{R} 2=0.9996)$, respectively. The analysis was carried out for six different dilutions $(0.25 \%, 0.5 \%, 1 \%, 2.5 \%, 5 \%$, and $10 \%)$ of each extract. The obtained results show that the highest amount of phenols and flavonoids was demonstrated in the $10 \%$ water-glycerine herb extract. The lowest concentration of these compounds showed a waterglycerine extract obtained from $M$. sativa $L$. seeds at a concentration of $0.25 \%$. Similar trends were observed for the flavonoid content. The difference between the content of phenolic compounds in the herb compared to seeds at the highest concentration of $10 \%$ was around $90 \%$ and in concentration of $5 \%$ it was around $75 \%$. Taking into account the content of flavonoids the difference between the herb and seeds was around $15 \%$ in case of concentration of $10 \%$ and around $35 \%$ in concentration of $5 \%$. It was also observed that TPC and TFC increased in a dose-dependent manner for all the extracts tested (Table 1).

The next stage of our research was the assessment of the ability of extracts from seeds and herb to remove free radicals. Therefore, the antioxidant potential was assessed using the DPPH radical. To determine the

\begin{tabular}{|c|c|c|c|c|}
\hline \multirow{2}{*}{$\begin{array}{l}\text { Extract } \\
\text { concentration } \\
{[\%]}\end{array}$} & \multicolumn{2}{|c|}{ TPC [mg GA/g] } & \multicolumn{2}{|c|}{$\mathrm{TFC}[\mathrm{mg} \mathrm{Qu/g}]$} \\
\hline & Seeds & Herb & Seeds & Herb \\
\hline 0.25 & $2.48 \pm 0.22^{\mathrm{a}}$ & $3.52 \pm 0.56^{\mathrm{b}}$ & $0.65 \pm 0.05^{c}$ & $1.58 \pm 0.40^{d}$ \\
\hline 0.5 & $4.43 \pm 0.48^{b}$ & $9.46 \pm 0.39^{\mathrm{e}}$ & $1.36 \pm 0.04^{d}$ & $4.12 \pm 0.23^{b}$ \\
\hline 1.0 & $8.67 \pm 0.29^{9}$ & $12.58 \pm 0.4^{\mathrm{h}}$ & $3.55 \pm 0.43^{b}$ & $5.33 \pm 0.47^{f}$ \\
\hline 2.5 & $11.55 \pm 0.37^{\mathrm{h}}$ & $19.84 \pm 0.26^{\mathrm{j}}$ & $5.52 \pm 0.41^{f}$ & $9.47 \pm 0.42^{\mathrm{g}}$ \\
\hline 5.0 & $20.58 \pm 0.36^{j}$ & $36.62 \pm 0.43^{k}$ & $13.87 \pm 0.11^{i}$ & $20.84 \pm 0.71^{j}$ \\
\hline 10.0 & $37.60 \pm 1.82^{\mathrm{k}}$ & $73.5 \pm 0.53^{\prime}$ & $21.35 \pm 0.63^{j}$ & $43.38 \pm 0.65^{m}$ \\
\hline
\end{tabular}

Values are mean of three replicate determinations $(n=3) \pm$ SD. Values not sharing the same letter are significantly different at $p<0.05$.

GA, gallic acid; TFC, total flavonoid content; TPC, total phenol content. 
antioxidant properties of the extracts tested, six different concentrations were used spanning from $0.25 \%$ to $10 \%$. Measurements were taken every $5 \mathrm{~min}$ in $30 \mathrm{~min}$. Based on the obtained data, it was shown that each extract has a different ability to reduce free radicals.

The highest ability of the DPPH radical scavenging was shown in the water-glycerine extract from the seeds at the highest concentration tested equal $10 \%$. After $30 \mathrm{~min}$ of the test the level of reduced DPPH• reached almost $40 \%$, while at the lowest concentration of $0.25 \%$ the level of scavenged radicals was about $3 \%$. In the case of herb, the level of scavenging of free radicals was significantly lower than in case of seeds in all concentrations tested, and at the highest concentration tested equal $10 \%$ and after $30 \mathrm{~min}$ it was about $20 \%$. In both analyzed raw materials, a correlation between the concentration and antioxidant potential of the extracts was also observed-higher the concentration used higher the force of free radicals reduction (Figs. 1 and 2).

An extremely important aspect in assessing the safety and toxicity of plant extracts is to evaluate their impact on cell metabolism and viability. In this study, we focused on the evaluation of cytotoxic properties of $M$. sativa L. extracts on two cell lines-fibroblasts (BJ) and keratinocytes ( $\mathrm{HaCaT})$. The tests used in the study (Neutral Red assay and Alamar Blue assay) allow to assess the degree of inhibition of growth related to concentration of the examined $M$. sativa $\mathrm{L}$. extracts. The results of the conducted experiments using Neutral Red assay indicate the positive effect of alfalfa extracts on the viability of both analyzed cell lines. This effect differs between fibroblasts and keratinocytes and is closely related to the concentration used. It was observed that these extracts exert a stronger effect on the viability of $\mathrm{HaCaT}$ than $\mathrm{BJ}$ cells, resulting in a greater increase in viability of these cells. The studies also showed that a slightly better effect is caused by the extract obtained from alfalfa herb in comparison with the seed extract. Alfalfa herb extract caused a slightly greater increase in keratinocytes viability to over $170 \%$ (at a concentration of $2.5 \%$ ), whereas in the case of seed extract this value reached less than $160 \%$ (at a concentration of 5\%). In the case of fibroblasts, the use of the highest concentrations of $M$. sativa L. herb extract resulted in an increase in fibroblast viability to about $150 \%$, while the seed extract increased the proliferation of these cells to less than $130 \%$ (at a concentration of 5\%) (Figs. 3 and 4).

Similar results were obtained using the Alamar Blue assay. The amount of resazurin reduced by the examined cells allowed a quantitative assessment of cell viability. This test also demonstrated that both alfalfa

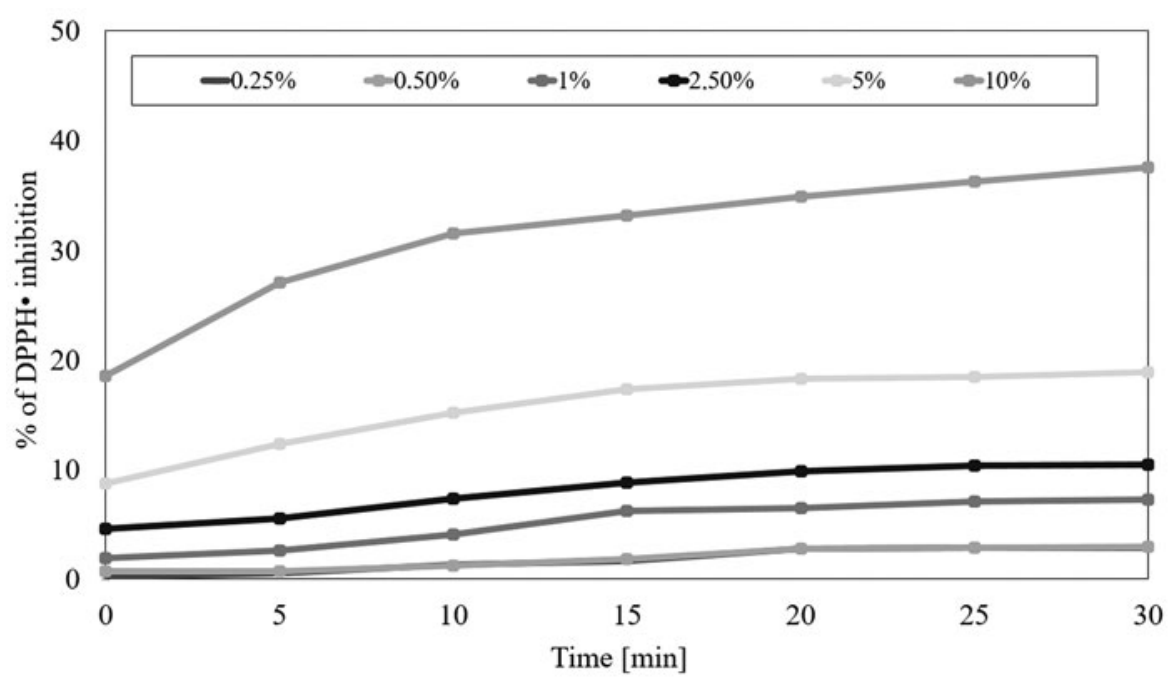

FIG. 1. Kinetics of the absorbance changes in DPPH. solutions in the presence of various concentrations of water-glycerine extract of Medicago sativa L. seeds. Values are mean of three replicate determinations $(n=3)$. DPPH, 2,2-diphenyl-1-picrylhydrazyl. 


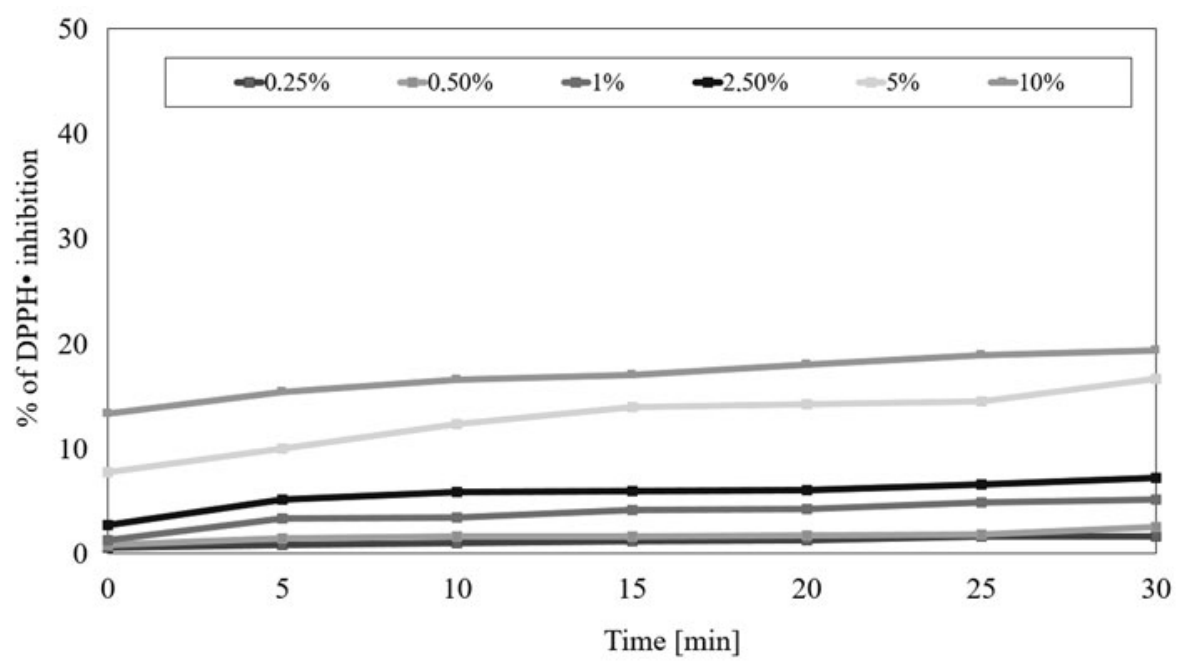

FIG. 2. Kinetics of the absorbance changes in DPPH - solutions in the presence of various concentrations of water-glycerine extract of M. sativa L. herb. Values are mean of three replicate determinations $(n=3)$.

seeds and herb UAE extracts have a positive effect on the viability of both cell lines. However, the differences observed between fibroblasts and keratinocytes were lower than those observed on the first test. The results of the analyzes performed with the use of this assay showed that the seeds extract strongly stimulates the viability of fibroblasts than keratinocytes and this increase reaches up to $130 \%$ (in the case of the three highest concentrations analyzed). Incubation of cells with extracts obtained from alfalfa herb resulted in an

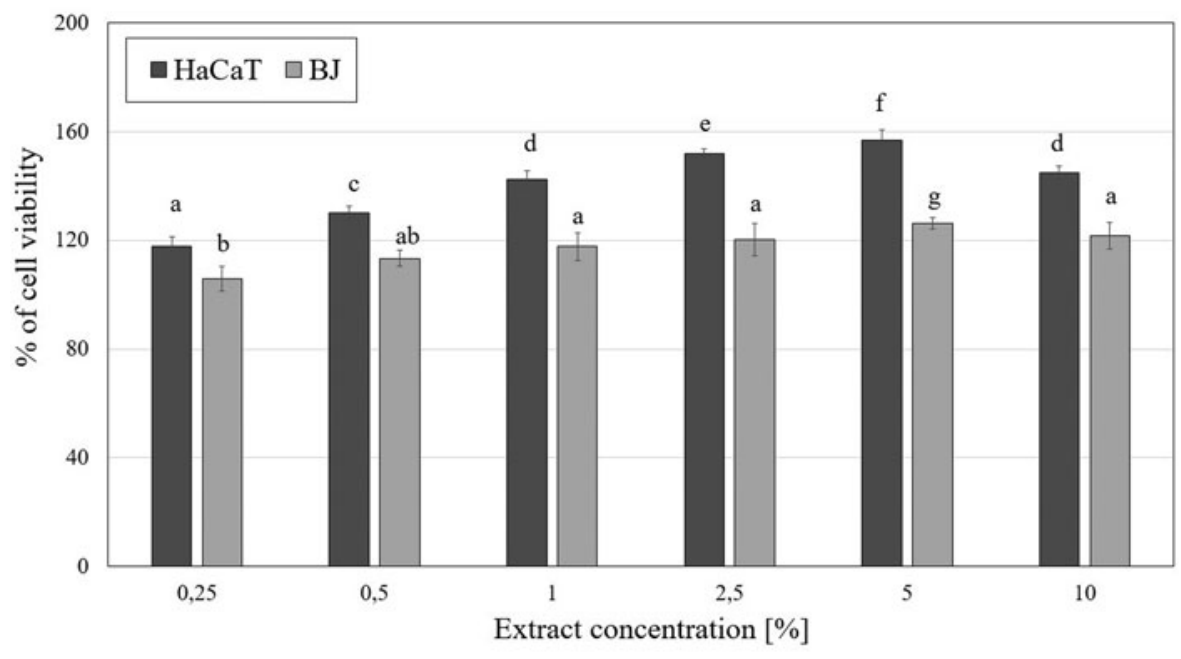

FIG. 3. The effect of increasing concentrations of $M$. sativa L. seeds extract (0.25-10\%) on Neutral Red Dye uptake in cultured keratinocytes ( $\mathrm{HaCaT}$ ) and fibroblasts (BJ) after $24 \mathrm{~h}$ of exposure. Data are the mean \pm SD of three independent experiments, each of which consists of three replicates per treatment group. Values not sharing the same letter are significantly different at $p<0.05$. 


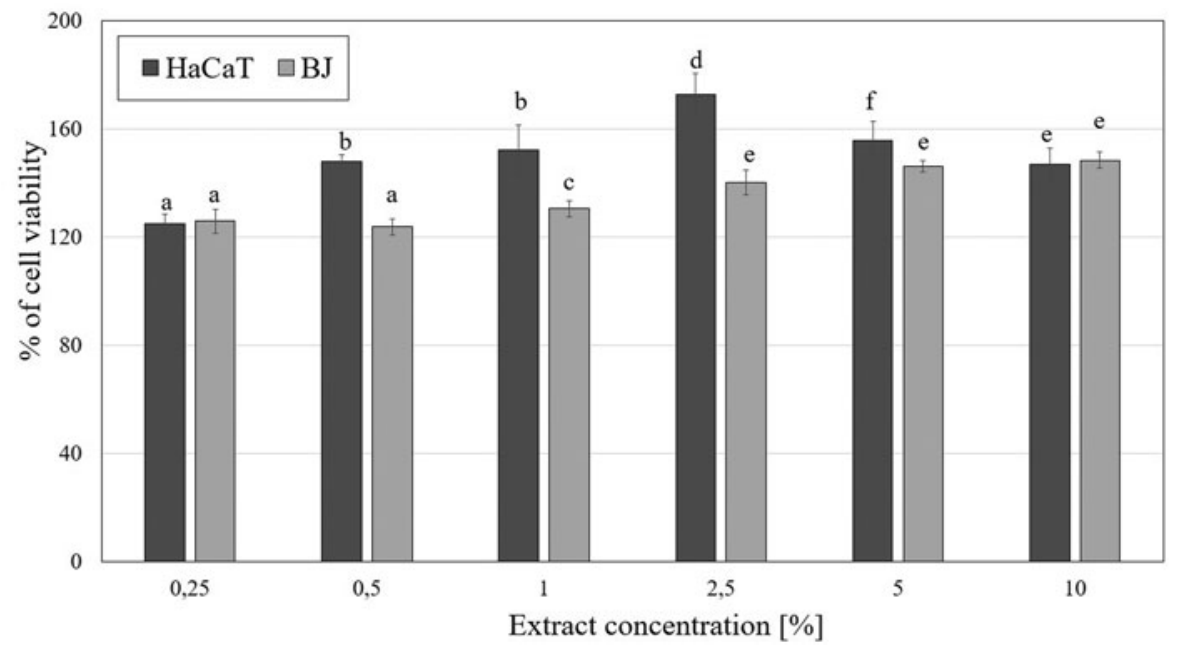

FIG. 4. The effect of increasing concentrations of $M$. sativa L. herb extract (0.25-10\%) on Neutral Red Dye uptake in cultured keratinocytes $(\mathrm{HaCaT})$ and fibroblasts (BJ) after $24 \mathrm{~h}$ of exposure. Data are the mean $\pm \mathrm{SD}$ of three independent experiments, each of which consists of three replicates per treatment group. Values not sharing the same letter are significantly different at $p<0.05$.

increase in $\mathrm{HaCaT}$ cells viability up to $120 \%$, whereas in the case of BJ cells no significant increase in viability was observed (Figs. 5 and 6).

To detect a disturbed redox balance in examined cells after exposure to $M$. sativa L. extracts the fluoro- genic dye $\mathrm{H}_{2}$ DCFDA was applied. This is one of the most commonly used probe for detecting intracellular ROS and oxidative stress. ${ }^{29}$ Before starting measurements using cell lines, we examined whether seeds and herb alfalfa UAE extracts without cells affected the

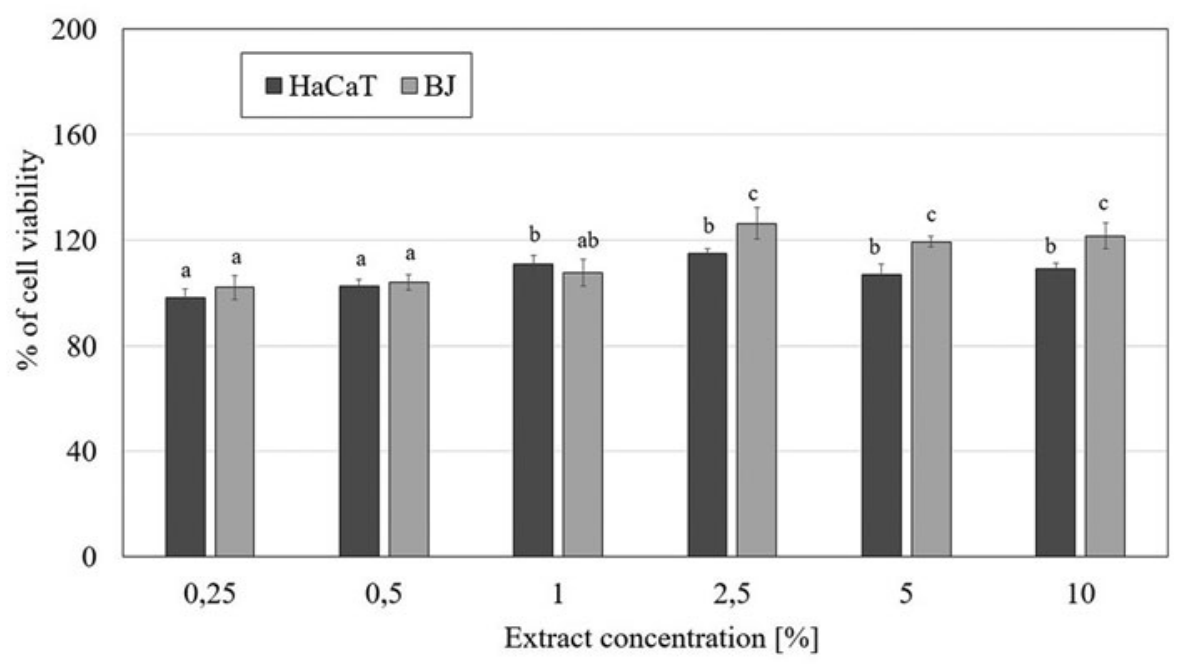

FIG. 5. The reduction of resazurin after $24 \mathrm{~h}$ exposure to the $M$. sativa $\mathrm{L}$. seeds extract $(0.25-10 \%)$ in cultured keratinocytes (HaCaT) and fibroblasts (BJ). Data are the mean \pm SD of three independent experiments, each of which consists of three replicates per treatment group. Values not sharing the same letter are significantly different at $p<0.05$. 


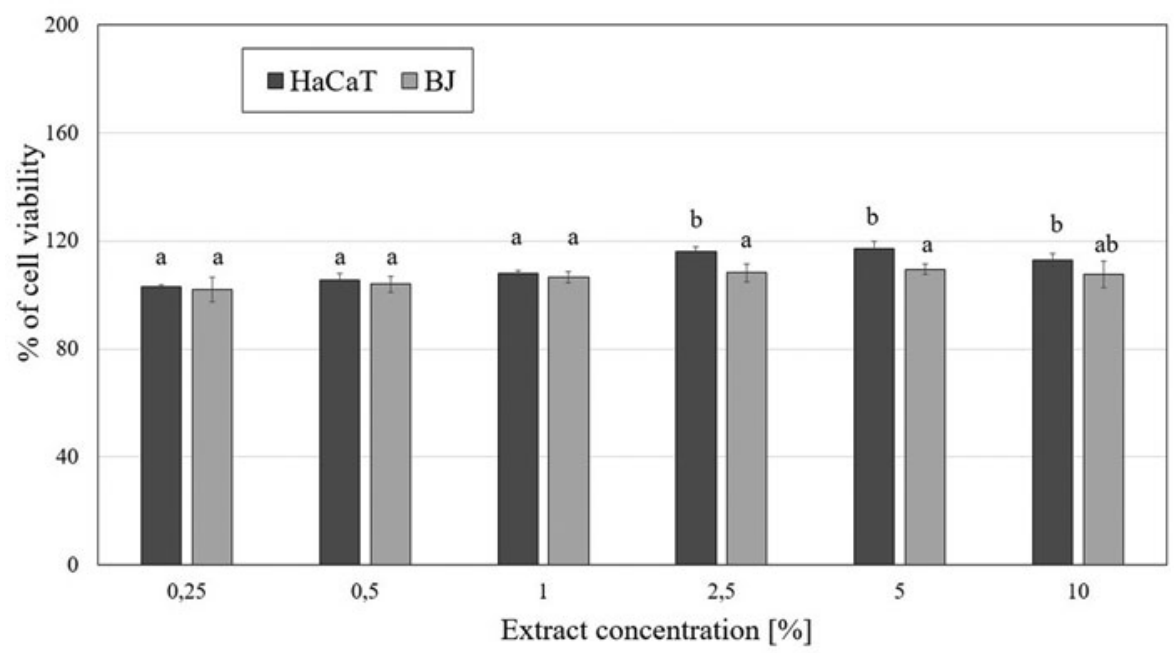

FIG. 6. The reduction of resazurin after $24 \mathrm{~h}$ exposure to the $M$. sativa $\mathrm{L}$. herb extract $(0.25-10 \%)$ in cultured keratinocytes $(\mathrm{HaCaT})$ and fibroblasts (BJ). Data are the mean $\pm \mathrm{SD}$ of three independent experiments, each of which consists of three replicates per treatment group. Values not sharing the same letter are significantly different at $p<0.05$.

fluorescence of the $\mathrm{H}_{2}$ DCFDA. Additionally, the separated experiment showed that there were no interactions between examined extracts and $\mathrm{H}_{2}$ DCFDA substrate in DMEM and MEM medium. The tests carried out showed that examined extracts can generate intracellular ROS in time- and dose-dependent manner. The activity of tested M. sativa L. extracts was also specific to the cell model. HaCaT cells treated with extracts in the concentration range from $0.25 \%$ to $10 \%$ exhibited correlation between used dose and intracellular free ROS level. After incubation of these cells with individual concentrations of extracts (both from the herb and seeds), an increase in the number of ROS was noted only at the highest concentration used (10\%). When alfalfa extracts were used in concentrations from $0.25 \%$ to $5 \%$, a decrease in intracellular ROS level was observed for both types of extracts (Figs. 7 and 8).

Analogous results were obtained when measuring the intracellular level of ROS in fibroblasts. As in the case of keratinocytes, the highest concentration of analyzed extracts increased the level of ROS. The values obtained after exposure of BJ cells to lower concentrations of both types of extracts fluctuated below the value obtained for the control (cells not exposed to the extract) or were significantly lower, which may indicate a decrease in intracellular ROS production significantly. Only after the fibroblasts were treated with the water-glycerine extracts of $M$. sativa $\mathrm{L}$. herb at a concentration of $0.25 \%$, the level of ROS oscillated slightly above the value obtained for the control (Figs. 9 and 10).

\section{Discussion}

Plant raw materials, with a large amount of biologically active substances can affect the activity and metabolism of cells. The main group of such substances includes polyphenolic compounds, which are one of the functional plant substances. They are characterized by strong antioxidant, anti-inflammatory, antifungal, and antiallergic properties. ${ }^{30-32}$ Chemical structure, type, and a level of the oxidation of the substrate and the conditions under which the oxidation process took place influence their activity. ${ }^{22,33}$ Both seeds and leaves are rich in compounds having antioxidant properties such as kaempferol, quercetin, luteolin, silbenes, coumarins, and others. ${ }^{34,35}$

The experiments carried out in this article clearly showed that the extract of alfalfa herb is a much richer source of biologically active substances compared with the extract obtained from the seeds of this plant. Thus, the herbal extract shows greater potential and may be beneficial in many aspects.

As mentioned above, the essential properties of phenolic compounds derived from plant material are their 


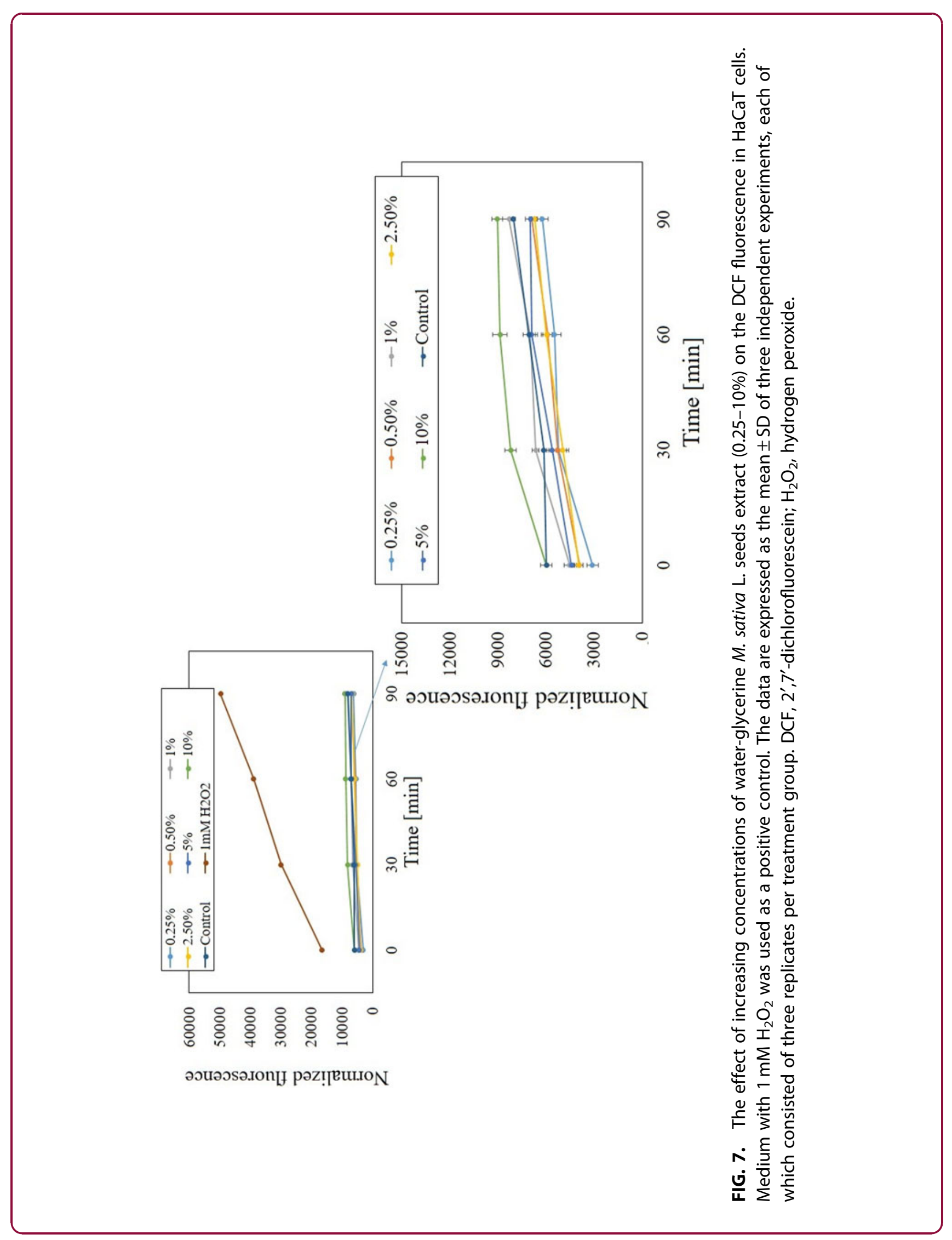




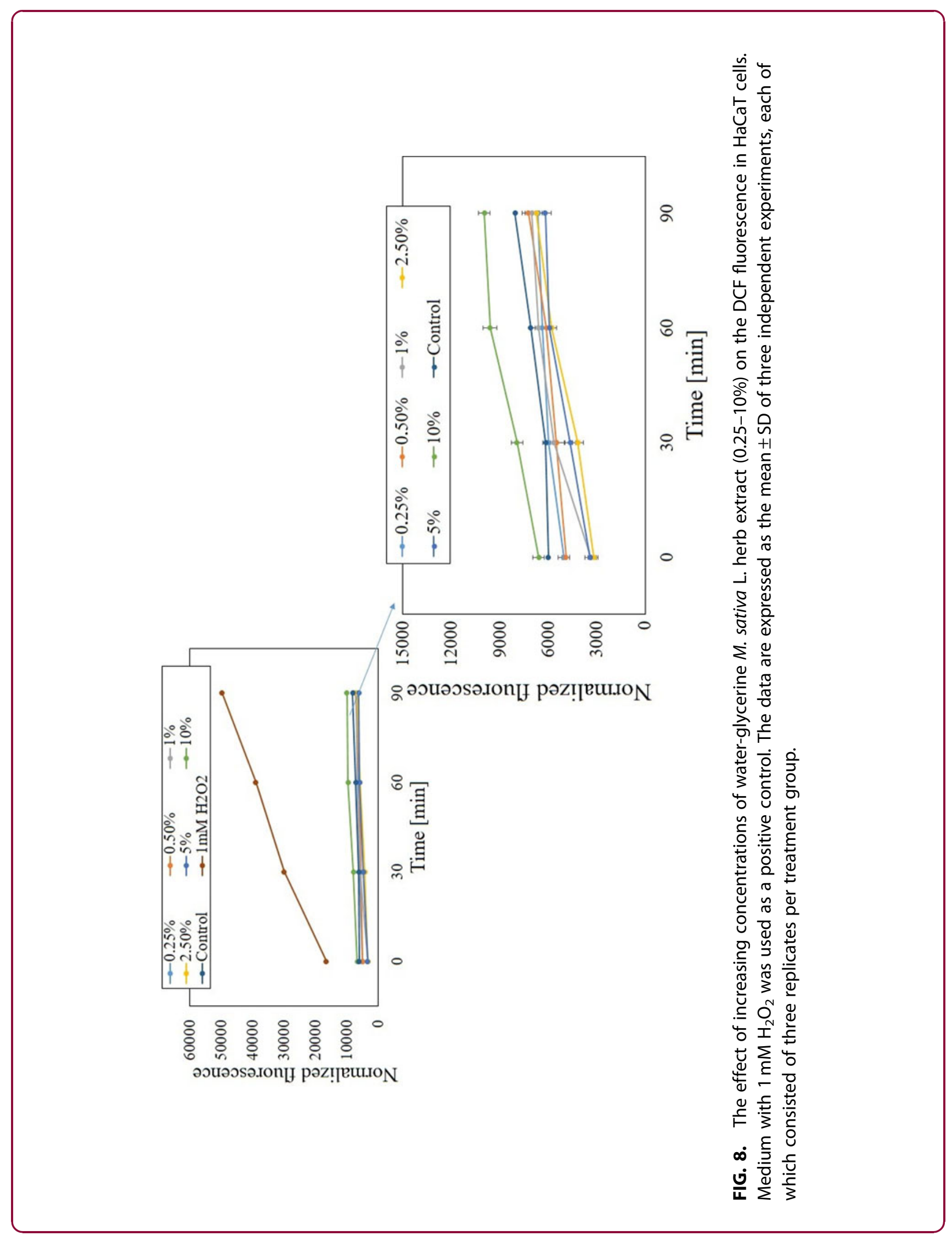




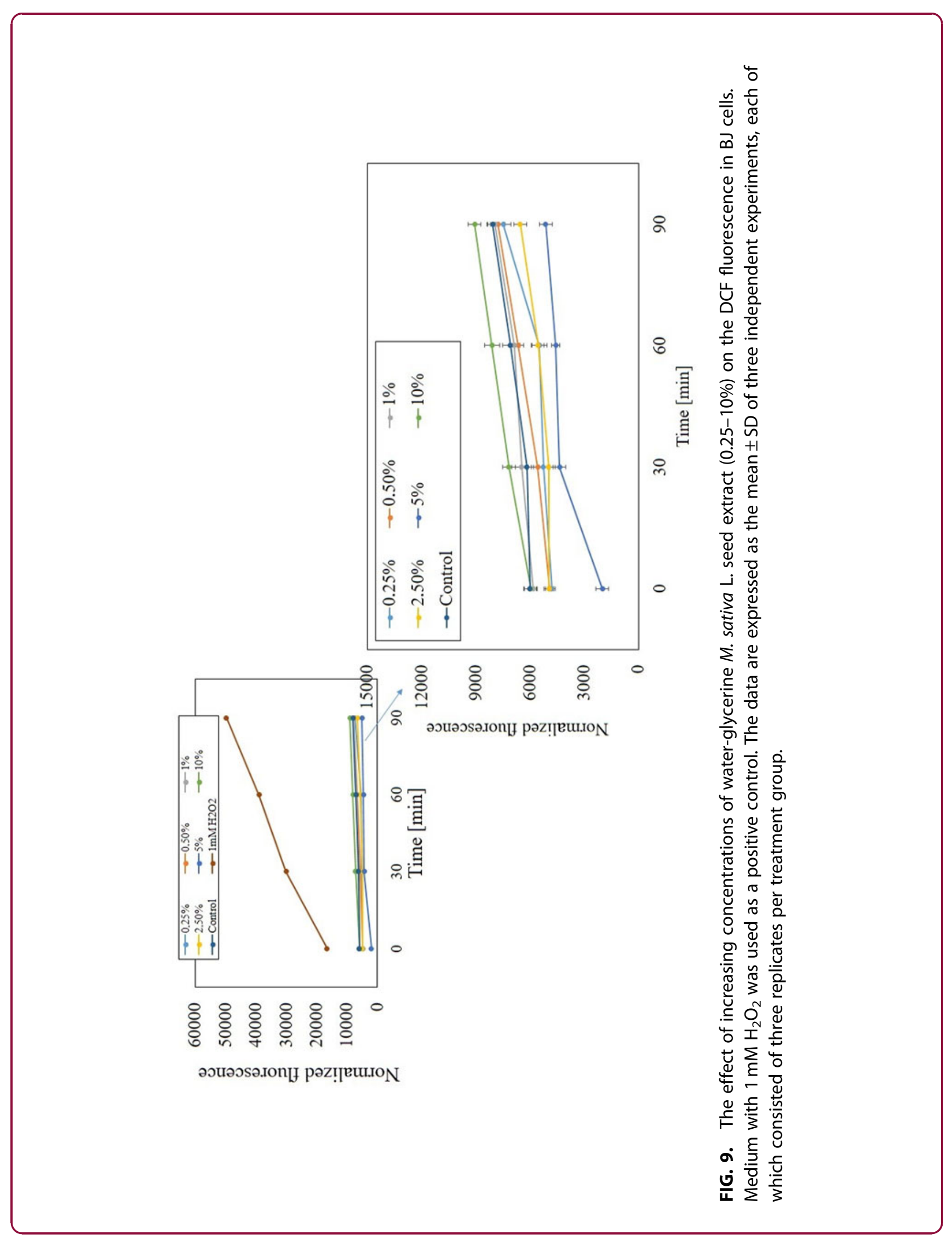




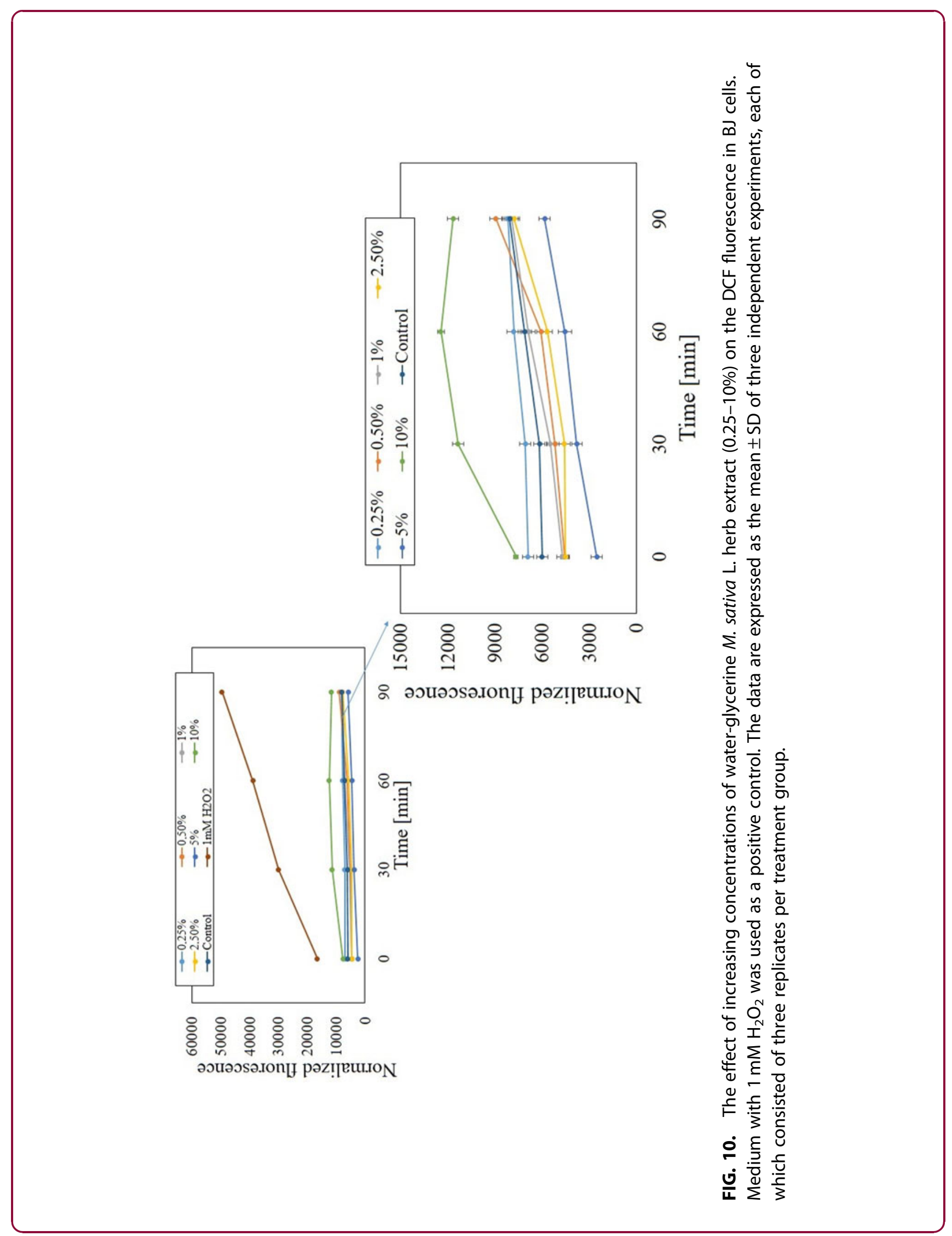


antioxidant properties. These compounds consist of a hydroxyl group and play a major role in the antioxidant capacity thanks to hydrogen releasing and the formation of stable radical intermediates. ${ }^{32}$ The mechanism of their action consists mainly of neutralization of free radicals, enzyme induction, and chelation of metal ions. ${ }^{21,36}$

The results of the DPPH• scavenging test obtained in this article correspond with other studies. Kudan and Anupam showed strong antioxidant activity of extracts obtained from $M$. sativa $\mathrm{L} .{ }^{35}$ High antioxidant activity was caused by a significant content of phenolic compounds, in particular quercetin or kaempferol. According to Rana et al. the extracts obtained from the roots of M. sativa $\mathrm{L}$ also show strong antioxidant activity. ${ }^{22}$

The cytotoxicity studies carried out in the next stage using the Alamar Blue and Neutral Red assays showed a beneficial effect of alfalfa extracts on the analyzed skin cells, both $\mathrm{HaCaT}$ and BJ. Although the results of both tests differ slightly, both clearly indicate that the extracts, both from the herb and seeds of $M$. sativa L., have a positive effect on the viability of fibroblasts and keratinocytes. The results obtained in these studies for the first time indicate the lack of toxicity of alfalfa extracts against skin cells and may suggest the potential use of these extracts in the pharmacological, dermatological, and cosmetic industries. In addition, the extracts obtained from this plant can be helpful in the fight against cancer, because experiments carried out by other researchers indicate their cytotoxic effects on several sensitive and multidrug-resistant tumor cells lines. ${ }^{37}$

As mentioned above, there are many articles indicating the antioxidant properties of alfalfa. ${ }^{21,22,35,36}$ As part of this work, these properties were also confirmed for the herb and seeds extracts of $M$. sativa $\mathrm{L}$. However, until now, there have been no studies assessing the effect of alfalfa extracts on the level of ROS inside cells treated with these extracts. Thus, the results of the conducted experiments indicate that the $M$. sativa $\mathrm{L}$. extracts not only affect the scavenging of free radicals existing in the external environment of the cell, but also have the ability to reduce the intracellular ROS level, which may contribute to the reduction of oxidative stress inside the cells. Due to the fact that oxidative stress leads to numerous intracellular disorders ${ }^{38}$ the exposure of skin cells to alfalfa extracts can have a positive effect.

\section{Conclusion}

The article was an attempt to determine the properties of extracts from seeds and herb of $M$. sativa $\mathrm{L}$. The tested extracts were characterized by a high content of biologically active phenolic compounds and flavonoids. It has been shown that the extracts have an antioxidant potential. It was also noted that tested extracts have a positive effect on the viability and proliferation of skin cells. The beneficial properties of alfalfa extracts shown in this article indicate the potential possibility of their use in the production of various types of cosmetic and pharmaceutical preparations applied to the skin. Although preliminary in vitro cytotoxicity studies on cells that are part of the various layers of the skin indicate no cytotoxicity, there is a need for further in vivo studies using animal models and clinical trials. Conducting this type of research will allow to assess the full safety of M. sativa L. extracts, which show promising properties in in vitro conditions.

\section{Author Disclosure Statement}

No competing financial interests exist.

\section{Funding Information}

This work was funded by the University of Information Technology and Management in Rzeszow, Poland.

\section{References}

1. Blanpain C, Fuchs E. Epidermal stem cells of the skin. Annu Rev Cell Dev Biol. 2006;22:339-373.

2. Kazanci A, Kurus $M$, Atasever A. Analyses of changes on skin by aging. Skin Res Technol. 2016;23:48-60.

3. Krutmann J, Bouloc A, Sore G, et al. The skin aging exposome. J Dermatol Sci. 2017;85:152-161.

4. Mora Huertas AC, Schmelzer CE, Hohenwarter W, et al. Molecular-level insights into aging processes of skin elastin. Biochimie. 2016;128-129: 163-173.

5. Devasagayam TPA, Tilak JC, Boloor KK, et al. Review: free radicals and antioxidants in human health: current status and future prospects. J Assoc Phys India. 2004;52:794-804.

6. Hunt JV, Dean RT, Wolff SP. Hydroxyl radical production and autoxidative glycosylation. Glucose autoxidation as the cause of protein damage in the experimental glycation model of diabetes mellitus and ageing. Biochem J. 1988;256:205-212.

7. Casaril M, Corso F, Corrocher R. Free radicals in human pathology. Recenti Prog Med. 1991;82:39-44.

8. Kancheva VD, Kasaikina OT. Bio-antioxidants-a chemical base of their antioxidant activity and beneficial effect on human health. Curr Med Chem. 2013;20:784-805.

9. Nimse SB, Pal D. Free radicals, natural antioxidants, and their reaction mechanisms. RSC Adv. 2015;5:27986.

10. Lü JM., Lin PH, Yao Q, et al. Chemical and molecular mechanisms of antioxidants: experimental approaches and model systems. J Cell Mol Med. 2010;14:840-860.

11. Almeida IF, Valentão $P$, Andrade $P B$, et al. Oak leaf extract as topical antioxidant: free radical scavenging and iron chelating activities and in vivo skin irritation potential. Biofactors. 2008;33:267-279.

12. Gomes-Rochette NF, Da Silveira Vasconcelos M, Nabavi SM, et al. Fruit as potent natural antioxidants and their biological effects. Curr Pharm Biotechnol. 2016;17:986-993.

13. Reichling J, Schnitzler P, Suschke U, et al. Essential oils of aromatic plants with antibacterial, antifungal, antiviral, and cytotoxic properties an overview. Forsch Komplementmed. 2009;16:79-90.

14. Miguel MG. Antioxidant and antiinflammatory activities of essential oils: a short review. Molecules. 2010;15:9252-9287. 
15. Orzechowski $A$, Ostaszewski $P$, Jank $M$, et al. Bioactive substances of plant origin in food-impact on genomics. Reprod Nutr Dev. 2002;42:461-477.

16. Zhao $Y, W u$ YZ, Wang M. Bioactive substances of plant origin. In: Handbook of Food Chemistry. Cheung P, Mehta B, (eds). Springer: Heidelberg, Berlin; pp. 1-35; 2015.

17. Butler H. Poucher's Perfumes, Cosmetics and Soaps, 10th ed. Kluwer Academic Publishers: London, 2000.

18. Aburjai T, Natsheh FM. Plants used in cosmetics. Phytother Res. 2003;17: 987-1000.

19. Karimi E, Oskouian E, Oskouian A, et al. Insight into the functional and medicinal properties of Medicago sativa (Alfalfa) leaves extract. J Med Plant Res. 2013;7:290-297.

20. Caunii A, Pribac G, Grozea I, et al. Design of optimal solvent for extraction of bio-active ingredients from six varieties of Medicago sativa. Chem Cent J. 2012;6:123.

21. Bora KS, Sharma A. Phytochemical and pharmacological potential of Medicago sativa: a review. Pharm Biol. 2011;49:211-220.

22. Rana MG, Katbamna RV, Padhya AA, et al. In vitro antioxidant and free radical scavenging studies of alcoholic extract of Medicago sativa L. Rom J Biol Plant Biol. 2010;55:15-22.

23. Fantini $M$, Benvenuto $M$, Masuelli $L$, et al. In vitro and in vivo antitumoral effects of combinations of polyphenols, or polyphenols and anticancer drugs: perspectives on cancer treatment. Int J Mol Sci. 2015;16:9236-9282.

24. Yang B, Liu X, Gao Y. Extraction optimization of bioactive compounds crocin, geniposide and total phenolic compounds from Gardenia Gardeniajasminoides Ellis fruits with response surface methodology. Innov Food Sci Emerg Technol. 2009;10:610-615.

25. Singleton VL, Orthofer R, Lamuela-Raventós RM. Analysis of total phenols and other oxidation substrates and antioxidants by means of folinciocalteu reagent. Method Enzymol. 1999;299:152-178.

26. Matejić JS, Džamić AM, Mihajlov-Krstev TM, et al. Total phenolic and flavonoid content, antioxidant and antimicrobial activity of extracts from Tordylium maximum. J Appl Pharm Sci. 2013;3:55-59.

27. Brand-Williams W, Cuvelier ME, Berset C. Use of a free radical method to evaluate antioxidant activity. LWT Food Sci Technol. 1995;28:25-30.

28. Borenfreund E, Puerner JA. Toxicity determined in vitro by morphological alterations and neutral red absorption. Toxicol Lett. 1985;24:119-124.

29. Dikalov SI, Harrison DG. Methods for detection of mitochondrial and cellular reactive oxygen species. Antioxid Redox Signal. 2014;20:372-382.

30. Velioglu YS, Mazza G, Gao L, et al. Antioxidant activity and total phenolics in selected fruits, vegetables, and grain products. J Agric Food Chem. 1998;46:4113-4117.

31. Halliwell B, Gutteridge JMC. Free Radicals in Biology and Medicine, 3rd ed. Oxford University Press: Oxford, 1999.
32. Alam MN, Bristi NJ, Rafiquzzaman M. Review on in vivo and in vitro methods evaluation of antioxidant activity. Saudi Pharm J. 2013;21:143-152.

33. Fukumoto LR, Mazza G. Assessing antioxidant and prooxidant activities of phenolic compounds. J Agric Food Chem. 2000;48:3597-3604.

34. Xue-Gui L, Ming-Yuan H, Pin-Yi G, et al. Bioactive constituents from Medicago sativa $\mathrm{L}$. with antioxidant, neuroprotective and acetylcholinesterase inhibitory activities. J Funct Foods. 2018;415:371-380.

35. Kudan SB, Anupam S. In vitro antioxidant and free radical scavenging potential of Medicago sativa Linn. J Pharm Res. 2010;3:1206-1210.

36. Zhao WS, Zhang YQ, Ren LJ, et al. Immunopotentiating effects of polysaccharides isolated from Medicago sativa L. (Abstract). Chung Kuo Yao Li Hsueh Pao. 1993;14:273-276.

37. Gatouillat G, Magid AA, Bertin E, et al. Cytotoxicity and apoptosis induced by alfalfa (Medicago sativa) leaf extracts in sensitive and multidrugresistant tumor cells. Nutr Cancer. 2014;66:483-491.

38. Alfadda AA, Sallam RM. Reactive oxygen species in health and disease. J Biomed Biotechnol. 2012;2012:936486.

Cite this article as: Zagórska-Dziok M, Ziemlewska A, NiziołŁukaszewska Z, Bujak T (2020) Antioxidant activity and cytotoxicity of Medicago sativa L. seeds and herb extract on skin cells, BioResearch Open Access 9:1, 229-242, DOI: 10.1089/biores.2020.0015.

Abbreviations Used
$\mathrm{DCF}=2^{\prime}, 7^{\prime}$-dichlorofluorescein
$\mathrm{DMEM}=$ Dulbecco's modified essential medium
$\mathrm{DPPH}=2,2$-diphenyl-1-picrylhydrazyl
$\mathrm{FBS}=$ fetal bovine serum
$\mathrm{GA}=$ gallic acid
$\mathrm{H}_{2} \mathrm{O}_{2}=$ hydrogen peroxide
$\mathrm{MEM}=$ Minimum Essential Medium
$\mathrm{PBS}=$ phosphate-buffered saline
$\mathrm{ROS}=$ reactive oxygen species
$\mathrm{TFC}=$ total flavonoid content
$\mathrm{TPC}=$ total phenol content
$\mathrm{UAE}=$ ultrasound-assisted extraction method
$\mathrm{UV}=$ ultraviolet

